\title{
A compact high-resolution elastic recoil detection system for lithium depth profiling
}

$\operatorname{AUTHOR}(\mathrm{S})$ :

Nikko, Masataka; Nakajima, Kaoru; Kimura, Kenji

\section{CITATION:}

Nikko, Masataka ... [et al]. A compact high-resolution elastic recoil detection system for lithium depth profiling. Nuclear Instruments and Methods in Physics Research Section B: Beam Interactions with Materials and Atoms 2015, 354: 240-243

ISSUE DATE:

2015-07-01

URL:

http://hdl.handle.net/2433/200805

\section{RIGHT:}

(c) 2015. This manuscript version is made available under the CC-BY-NC-ND 4.0 license

http://creativecommons.org/licenses/by-nc-nd/4.0/; The full-text file will be made open to the public on 1 July 2017 in accordance with publisher's 'Terms and Conditions for Self-Archiving'.; この論文は出版社版でありません。引用の際に は出版社版をご確認ご利用ください。; This is not the published version. Please cite only the published version. 


\title{
A compact high-resolution elastic recoil detection system for lithium depth profiling
}

\author{
Masataka Nikko, Kaoru Nakajima and Kenji Kimura* \\ ${ }^{1}$ Department of Micro Engineering, Kyoto University, Kyoto 615-8540, Japan \\ *e-mail: kimura@kues.kyoto-u.ac.jp
}

A compact high-resolution elastic recoil detection analysis (ERDA) system was developed for precise $\mathrm{Li}$ analysis. $200-400 \mathrm{keV} \mathrm{He}^{+}$ions were used as primary ions and the energy spectra of recoiled Li ions were measured using a magnetic spectrometer. Due to its dispersion the background originating from the scattered primary ions is reduced. The recoiled ions of the substrate other than $\mathrm{Li}$, which may also contribute to the background, were rejected by a thin mylar foil placed in front of a focal plane detector. An electrostatic quadrupole lens was installed at the entrance of the magnetic spectrometer to improve the depth resolution by correcting the effect of the kinematic broadening. The performance of the developed high-resolution ERDA was examined by measuring several samples. A thin Li layer $\left(1.5 \times 10^{15} \mathrm{~cm}^{-2}\right)$ deposited on a graphite surface was clearly observed and the detection limit was estimated to be less than 0.01 ML under typical measurement conditions. The depth resolution was estimated to be $0.5 \mathrm{~nm}$ at the surface and was better than $3 \mathrm{~nm}$ in the surface region within $5 \mathrm{~nm}$ from the surface. 


\section{Introduction}

Lithium ion batteries are widely used as power sources for portable electronics, electric vehicles etc. Because intercalation of lithium into the electrodes is one of the key processes in the $\mathrm{Li}$ ion batteries, understanding of the intercalation mechanism is of prime importance to improve battery performance [1]. To this end, precise analysis of Li at the electrode surfaces is required. Elastic recoil detection analysis (ERDA) is one of the powerful techniques for depth profiling of light elements. Dollinger et al developed a high-resolution ERDA with a sub-nm depth resolution using a sophisticated magnetic spectrograph $[2,3]$. This allows depth profiling of light elements with a depth resolution of $\sim 0.5 \mathrm{~nm}$ at the surface and with a sensitivity down to the $100 \mathrm{ppm}$ level. They used a rather large magnetic spectrograph originally designed for nuclear physics experiments [4] and a large tandem accelerator (14 MV tandem accelerator) to utilize high energy heavy ions, e.g. $100 \mathrm{MeV} \mathrm{Au} \mathrm{ions.} \mathrm{Although} \mathrm{the} \mathrm{performance} \mathrm{of} \mathrm{their} \mathrm{high-resolution} \mathrm{ERDA} \mathrm{is} \mathrm{excellent,}$ need of such a big facility prevents it from spreading into wide application fields. There was another approach to develop high-resolution ERDA using a relatively small accelerator (e.g. 2MV tandem accelerator) [5]. Vandervorst and his coworkers employed a time of flight (TOF) detection system in combination with relatively low energy ( $<20 \mathrm{MeV}$ ) heavy ions ( $\mathrm{Cl}$ ion, $\mathrm{Cu}$ ion etc). The achieved depth resolution, however, is several $\mathrm{nm}$, which may not be good enough for some applications. We have developed a compact high-resolution ERDA system for precise Li analysis with a sub-nm depth resolution using a small magnetic spectrometer and a small accelerator. The aim of the present paper is to describe the performance of the developed system.

\section{Rejection of scattered primary ions}

In ERDA, primary ions scattered from the specimen contribute to a background in the energy spectrum. In the conventional ERDA for hydrogen analysis, the scattered primary ions are rejected by a thin foil installed in front of a silicon surface barrier detector [6]. The foil, however, deteriorates the depth resolution due to the energy loss straggling of the recoiled ions during the passage through the foil. In the high-resolution ERDA, a 
position-sensitive ionization chamber which can measure the position and ion species simultaneously is used as a focal plane detector of the magnetic spectrograph. Using such a detector, the energy spectrum of recoiled ions of a specific element can be measured separately from other ions [3]. In the present ERDA system, however, such a focal plane detector cannot be used because the energies of the recoiled ions are only several hundred $\mathrm{keV}$ and far below the detection limit of such a detector. In order to reject the scattered primary ions, we employ He ion beam as a probe instead of heavy ions. When the magnetic field of the spectrometer is adjusted for the recoiled Li ions, scattered He ions are deflected at larger angles and cannot reach the focal plane detector. For example, when a $400 \mathrm{keV} \mathrm{He}$ beam is used as a primary beam the enrgy of $\mathrm{Li}$ ions recoiled at $25^{\circ}$ with respect to the incident beam is about $304 \mathrm{keV}$. If the magnetic field is adjusted to pass $304 \mathrm{keV} \mathrm{Li}^{+}$ions, both $532 \mathrm{keV} \mathrm{He}^{+}$and $2128 \mathrm{keV} \mathrm{He}^{2+}$ ions can pass through the magnetic spectrometer. However, the actual energies of the scattered He ions are, of course, lower than the primary energy, $400 \mathrm{keV}$. Thus there is no background originating from the scattered primary ions in the ERDA spectrum.

\section{Experimental}

A schematic drawing of our high-resolution ERDA setup is shown in Fig. 1. Beams of $200-400 \mathrm{keV} \mathrm{He}^{+}$ions were produced by a small accelerator and collimated by a couple of slit systems to $2 \times 2 \mathrm{~mm}^{2}$ in size. The beam current was monitored by a beam chopper installed just before the scattering chamber and a typical beam current was $\sim 50 \mathrm{nA}$. The collimated $\mathrm{He}^{+}$beam was sent to a scattering chamber (base pressure $2 \times 10^{-8} \mathrm{~Pa}$ ), where a sample was mounted on a 5-axis precision gomiometer. The ions recoiled by the $\mathrm{He}^{+}$ions at $25^{\circ}$ with an exit angle of $10^{\circ}$ with respect to the surface plane were analyzed by a $90^{\circ}$ sector magnetic spectrometer and detected by a one-dimensional position-sensitive detector (1D-PSD) consisting of Z-stack micro channel plates (MCPs) and a resistive anode (effective length $100 \mathrm{~mm})$. The energy dispersion $(E \mathrm{~d} x / \mathrm{d} E$, where $x$ is the position on the focal plane) of the spectrometer is $400 \mathrm{~mm}$ and the acceptance angle of the spectrometer is \pm 10 mrad both in the vertical and horizontal directions. Although the acceptance angle is not 
so large, a so-called kinematic broadening causes deterioration in the depth resolution. In the present case, the kinematic broadening is calculated to be $1.9 \%$ of the recoiled energy, which corresponds to a depth resolution of a few nm under typical experimental conditions. In order to correct the effect of the kinematical broadening, an electrostatic quadrupole lens (Q-lens) was installed between the sample and the spectrometer. Using the Q-lens, the $\mathrm{Li}^{+}$ ions recoiled from surface atoms at different angles within the acceptance angle can be foused on the same position in the energy dispersive plane of the detector.

\section{Result and discussion}

A highly oriented polycrystalline graphite (HOPG) was mounted on the goniometer. A small amount of Li was deposited in situ on the surface. The amount of Li was measured to be $1.5 \times 10^{15} \mathrm{~cm}^{-2}$ (corresponding to $0.4 \mathrm{ML}$ of graphite) using high-resolution Rutherford backscattering spectroscopy (RBS) after ERDA measurements. In the high-resolution RBS measurement, a different area was used to avoid a possible modification due to ERDA measurements. Figure 2 shows an example of the observed ERDA spectra for the Li-deposited graphite sample (solid circles) using a $200 \mathrm{keV} \mathrm{He}^{+}$beam. There is a small peak at $\sim 152 \mathrm{keV}$, which corresponds to the deposited surface Li. Although there should be no background originating from the scattered He ions as was mentioned above, there is in fact a large background. The origin of the observed background may be ascribed to the recoiled carbon ions. The recoiled $88 \mathrm{keV} \mathrm{C}^{+}$ions have the same trajectory as the $152 \mathrm{keV} \mathrm{Li}^{+}$ion in the magnetic spectrometers and these ions contribute to the observed background. In order to reject this large background, a mylar foil (thickness $0.5 \mu \mathrm{m}$ ) was installed in front of the 1D-PSD (9mm from the MCP entrance surface). A thin Al layer (20 nm) was deposited on the mylar surface to prevent possible charging. The thickness of the foil was chosen so that the projected range of $88 \mathrm{keV} \mathrm{C}^{+}\left(152 \mathrm{keV} \mathrm{Li}^{+}\right)$is shorter (longer) than the foil thickness. The passage of the Li ions through the foil hardly deteriorates the energy resolution of the spectrometer because the energy analysis of the recoiled Li ions has already been completed when the ion pass through the foil. The only possible deterioration comes from the angular spread due to multiple scattering in the foil. The angular distribution of Li ions passing 
through the mylar foil was calculated using the TRIM code [7]. The calculated FWHM is 0.3 and 0.13 rad for 152 and $304 \mathrm{keV} \mathrm{Li} \mathrm{ions,} \mathrm{respectively.} \mathrm{These} \mathrm{angular} \mathrm{spread} \mathrm{result} \mathrm{in}$ the spatial spread of 2.7 and $1.2 \mathrm{~mm}$, respectively, on the MCP surface, which corresponds to the energy resolution of 1.0 and $0.9 \mathrm{keV}$, respectively.

The observed ERDA spectrum with the mylar foil is shown by open circles in Fig. 2. The background was reduced by a factor of 100 and the Li peak is clearly seen. Note that there is a long tail in the low energy side of the Li peak, which cannot be seen without the foil. This tail indicates that a part of deposited $\mathrm{Li}$ atoms penetrate through the surface and distribute in the sub-surface region of a few $\mathrm{nm}$ from the surface. There is still a residual background, which is ascribed to the dark noise of the MCP $\left(\sim 0.07\right.$ counts $\mathrm{cm}^{-2} \mathrm{~s}^{-1}$ in the present case). By integrating the spectrum in the energy region from 145 to $154.5 \mathrm{keV}$, the total yield of the Li peak was calculated to be $~ 1100$ counts. The background yield in the corresponding region is $\sim 180$ counts. Thus the net counts of the Li signal is $900 \pm 36$ (= $\sqrt{ } 1100+180)$ in the present case $\left(1.5 \times 10^{15} \mathrm{Li}\right.$ atoms $\left.\mathrm{cm}^{-2}\right)$. If the detection limit is defined so that the net counts is equal to the statistical error, the detection limit of surface Li on a graphite surface is estimated to be $3 \times 10^{13} \mathrm{~cm}^{-2}$, which corresponds to $\sim 0.008 \mathrm{ML}$, under the present experimental conditions $(50 \mathrm{nA} \times 1000 \mathrm{~s})$. Note that the detection limit could be improved by increasing the measurement time because the net counts increases linearly with measurement time while the statistical error increases more slowly.

Figure 3 shows another examples of the observed ERDA spectrum using $400 \mathrm{keV}$ $\mathrm{He}^{+}$ions. The sample was prepared by vacuum evaporation of $\mathrm{LiF}$ on $\mathrm{Si}(001)$. The thickness of the LiF layer was measured to be $3 \mathrm{~nm}$ using high-resolution RBS. The open circles show the spectrum observed without Q-lens. From the slope of the leading edge, the full width at half maximum (FWHM) of the resolution function was estimated to be $5.2 \mathrm{keV}$. This is in good agreement with the calculated kinematic broadening (5.7 keV), indicating that the effective energy resolution is mainly determined by the kinematic broadening. Using the stopping powers, the depth resolution was calculated to be $1.7 \mathrm{~nm}$ at the surface. This is better than the depth resolution of the TOF-ERDA setup mentioned above but several times worse compared to the resolution achieved by Dollinger et al using high-energy heavy ions 
and the sophisticated magnetic spectrograph. In order to improve the depth resolution, trajectory simulations of the recoiled $\mathrm{Li}^{+}$ions were performed to determine the optimal voltage applied to the Q-lens. The ERDA spectrum observed with the Q-lens is shown by closed circles in Fig. 3. The leading edge becomes steeper compared to the result without the Q-lens, indicating improvement of the depth resolution. The leading edge was fitted to an error function. The obatined effective energy resolution at the surface is $1.6 \mathrm{keV}$, which represents the experimental energy resolution of the present setup. This energy resolution corresponds to the depth resolution of $0.5 \mathrm{~nm}$ at the surface. The obtained depth resolution is comparable to that achieved by Dollinger et al in spite of the much smaller equipment used in the present work.

Although the obtained depth resolution is excellent at the surface, it is known that the depth resolution deteriorates with depth due to the energy loss straggling as well as the multiple scattering. Actually the slope of the trailing edge ( $295 \mathrm{keV})$ is much more gentle compared to the leading edge, indicating that the depth resolution deteriorates at the interface of $\mathrm{LiF} / \mathrm{Si}$. In order to estimate the depth resolution in a deeper region, Monte Carlo simulation for the ERDA specrum was perforemd using the Corteo code [8]. Energy spectra of $\mathrm{Li}$ ions recoiled by $400 \mathrm{keV}$ He ions from a thin Li layer (areal density $5 \times 10^{13} \mathrm{Li}$ atoms $\mathrm{cm}^{-2}$ ) located at a depth $x$ in Si were calculated for various $x$. In the simulation, the input parameters (scattering geometry, the acceptance angle of the spectrometer etc) were chosen to be the same as the measurements shown in Fig. 3. Figure 4 shows examples of the calculated spectra. The spectrum for $x=0 \mathrm{~nm}$ shows a rectangular shape and the width of the spectrum (5.7 keV) agrees with the calculated kinematic broadening (5.7 keV), indicating that the depth resolution at the surface is determined mainly by the kinematic broadening. With increasing depth, the spectrum becomes broader due to the multiple scattering and the energy loss straggling. The FWHMs of the calculated spectra are shown by open circles in Fig. 5. Using the stopping powers, the obtained FWHM can be converted to the depth resolution, which is shown by the vertical axis in the right hand side. Note that the calculated spectrum includes the effect of the kinematic broadening while the effect can be corrected using the Q-lens in the present ERDA system. Thus the depth resolution of the 
present ERDA system is better than the result of the Corteo simulation.

The effect of the kinematic broadening was removed from the result of the Corteo simulation through devonvolution procedures. The resultant depth resolutions are shown by triangles in Fig. 5. Finally, taking account of the observed experimental energy resolution (1.6 keV), the depth resolution for the present setup was obtained. The obtained result was shown by closed circles in Fig. 5, which is about 0.5, 2 and $3 \mathrm{~nm}$ at $x=0,2$ and $5 \mathrm{~nm}$, respectively.

The ERDA spectrum for $\operatorname{LiF}(2.8 \mathrm{~nm}) / \mathrm{Si}$ calculated using the Corteo code is shown by a thin solid line in Fig. 3. Although the calculated spectrum agrees with the obaserved spectrum at the leading edge, there is a notable discripancy at the trailing edge. The observed trailing edge is slightly gentle compared to the calculated spectrum. This indicates that the LiF layer is not uniform. Assuming an appropriate thickness distribution, the observed spectrum can be reproduced by the Corteo simulation as shown by a thick solid line. The obtained thickness distribution is shown in the inset of Fig. 3, which indicates that the $\mathrm{LiF}$ layer is rather uniform.

Finnally we note that the information of the charge state distribution of recoiled Li ions is indispensable for quantitative analysis becuse only $\mathrm{Li}^{+}$ions can be measured in the present setup. The semi-empirical chrage state distribution of $\mathrm{Li}$ ions passing through a carbon foil was given by Marion and Young [9]. According to their result, the $\mathrm{Li}^{+}$fraction is larger than $60 \%$ in a energy region from 235 to $550 \mathrm{keV}$ with a maximim fraction of $66 \%$ at $400 \mathrm{keV}$. Although the charge state distribution may depends on the material, their result suggests that the $\mathrm{Li}^{+}$fraction is almost constant if we measure mainly the surface region using $400 \mathrm{keV} \mathrm{He}^{+}$as the probe like the measurement shown in Fig. 3.

\section{Conclusion}

A compact high-resolution ERDA system consisting of a small magnetic spectrometer and a small accelerator was developed for precise Li analysis. $200-400 \mathrm{keV}$ He ions were used as the probe. This allows complete rejection of the scattered He ions from the Li spectrum. The recoiled substrate ions were also rejected and the background is 
drastically reduced using a thin mylar foil placed in front of the focal plane detector. The residual background was almost the same level as the dark noise of MCP. The detection limit of Li was estimated to be less than 0.01 ML under a typical measurement conditions. The depth resolution at the surface was measured to be $0.5 \mathrm{~nm}$. The depth resolution in the deeper region was estimated by the Corteo simulation. The estimated depth resolution is 2 and $3 \mathrm{~nm}$ at $x=3$ and $5 \mathrm{~nm}$, respectively. These depth resolutions are comparable to that achieved by Dollinger et al using a large magnetic spectrograph and a large accelerator, although their depth resolution does not deteriorate that fast with depth and their setup is not restricted to $\mathrm{Li}$.

\section{Acknowledgement}

This work was supported by JSPS KAKENHI Grant Number 25286020. 


\section{References}

[1] J.-M. Tarascon and M. Armand, Nature 414 (2001) 359.

[2] G. Dollinger, C.M. Frey, A. Bergmaier, T. Faestermann, Europhys. Lett. 42 (1998) 25.

[3] G. Dollinger A. Bergmaier, L. Goergens, P. Neumaier, W. Vandervorst, S. Jakschik, Nucl. Instrum. Methods Phys. Res., Sect. B 219-220 (2004) 333. $117 . \quad$ 219-220(2004) 333-343.

[4] M. Löffler, H.-J. Scheerer, H. Vonach, Nucl. Instrum. Methods 111 (1973) 1.

[5] S. Giangrandi, T. Sajavaara, B. Brijs, K. Arstila, A. Vantomme, W. Vandervorst, Nucl. Instrum. Methods Phys. Res., Sect. B 266 (2008) 5144.

[6] J. L’ Ecuyer, C. Brassard, C. Cardinal, J. Chabbal, L. Deschenes, J.P. Labrie, B. Terreault, J.G. Martel, R.St. Jacques, J. Appl. Phys 47 (1976) 382.

[7] J.F. Ziegler, J.P. Biersack, U.L. Littmark, The Stopping and Range of Ions in Solids, Pergamon Press, New York, 1985.

[8] F. Schiettekatte, Nucl. Instrum. Methods Phys. Res., Sect. B 266 (2008) 1880.

[9] J.B. Marion, F.C. Young, Nuclear Reaction Analysis, Graphs and Tables, North-Holland, Amsterdam, 1968. 


\section{Figure captions}

Fig. 1 Schematic drawing of experimental setup for high-resolution ERDA.

Fig. 2 Examples of observed ERDA spectra for Li/graphite. The ions recoiled by the $\mathrm{He}^{+}$ ions at $25^{\circ}$ with an exit angle of $10^{\circ}$ with respect to the surface plane were measured when $200 \mathrm{keV} \mathrm{He}^{+}$ions were incident on the sample. The spectra observed with and without a mylar foil are shown by closed and open circles, respectively. The measurement time was $1000 \mathrm{~s}$ for both spectra.

Fig. 3 Examples of observed ERDA spectra for $\mathrm{LiF}(2.8 \mathrm{~nm}) / \mathrm{Si}(001)$. The ions recoiled by the $\mathrm{He}^{+}$ions at $25^{\circ}$ with an exit angle of $10^{\circ}$ with respect to the surface plane were measured when $400 \mathrm{keV} \mathrm{He}{ }^{+}$ions are incident on the sample. The spectra observed with and without Q-lens are shown by closed and open circles, respectively. The result of Corteo simulation for a uniform LiF layer of $2.8 \mathrm{~nm}$ thickness is shown by a thin solid line. The best fit result of Corteo simulation for a non-uniform LiF layer is also shown by a thick solid line. The inset shows the corresponding thickness distribution of the LiF layer.

Fig. 4 Energy spectra of Li ions recoiled from a thin Li layer (areal density $5 \times 10^{13}$ atoms $\mathrm{cm}^{-2}$ ) embedded in $\mathrm{Si}$ at various depths calculated using Corteo code. The incident ion, scattering geometry and the acceptance angle of the spectrometer are the same as the measurement shown in Fig. 3.

Fig. 5 The FWHMs of the Li peaks shown in Fig. 4 (open circles). The vertical axis in the right hand side shows the corresponding depth resolution. Estimated depth resolution after the correction of the kinematic broadening (triangles) and the result including the experimental energy resolution (closed circles) are also shown. 


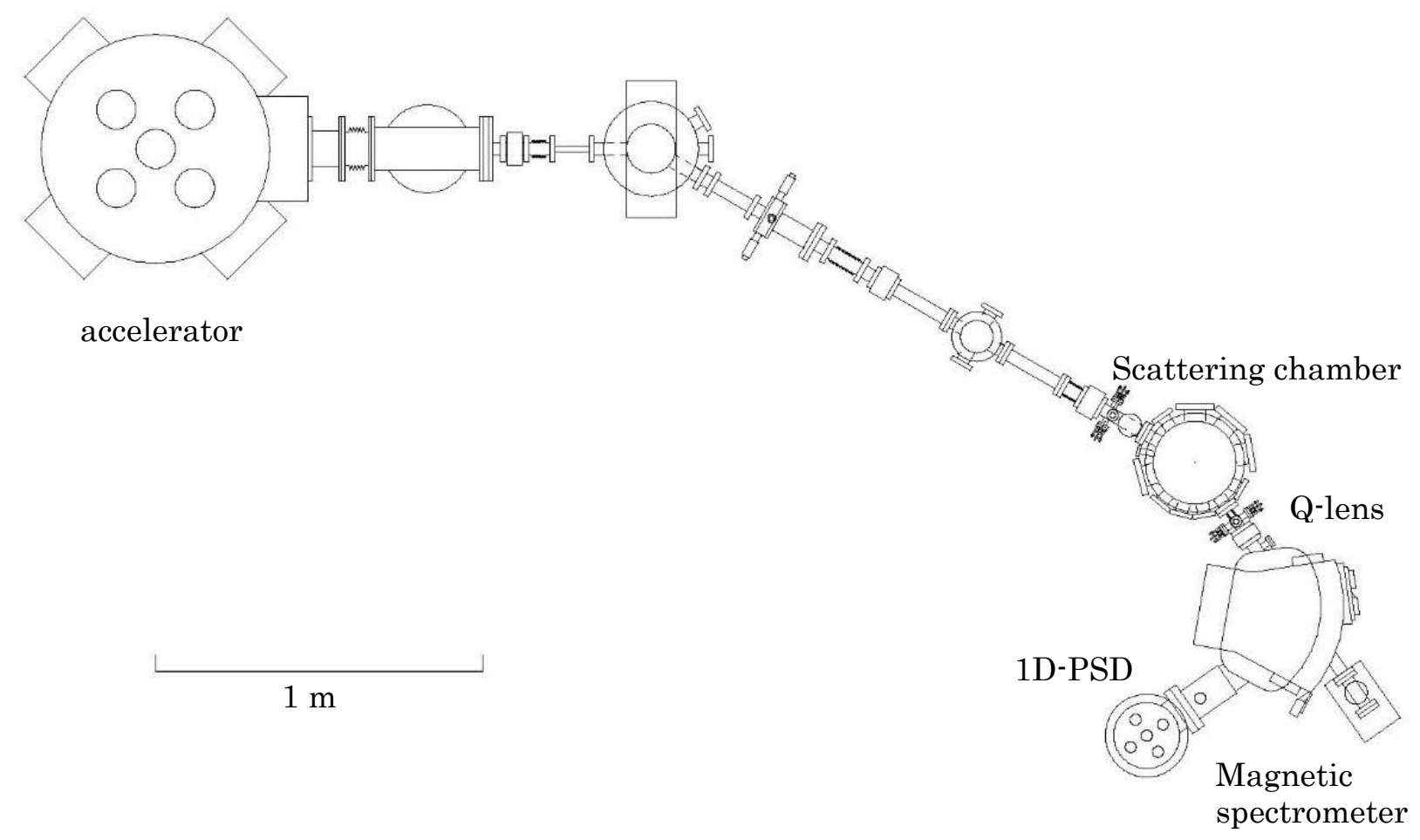

Fig. 1

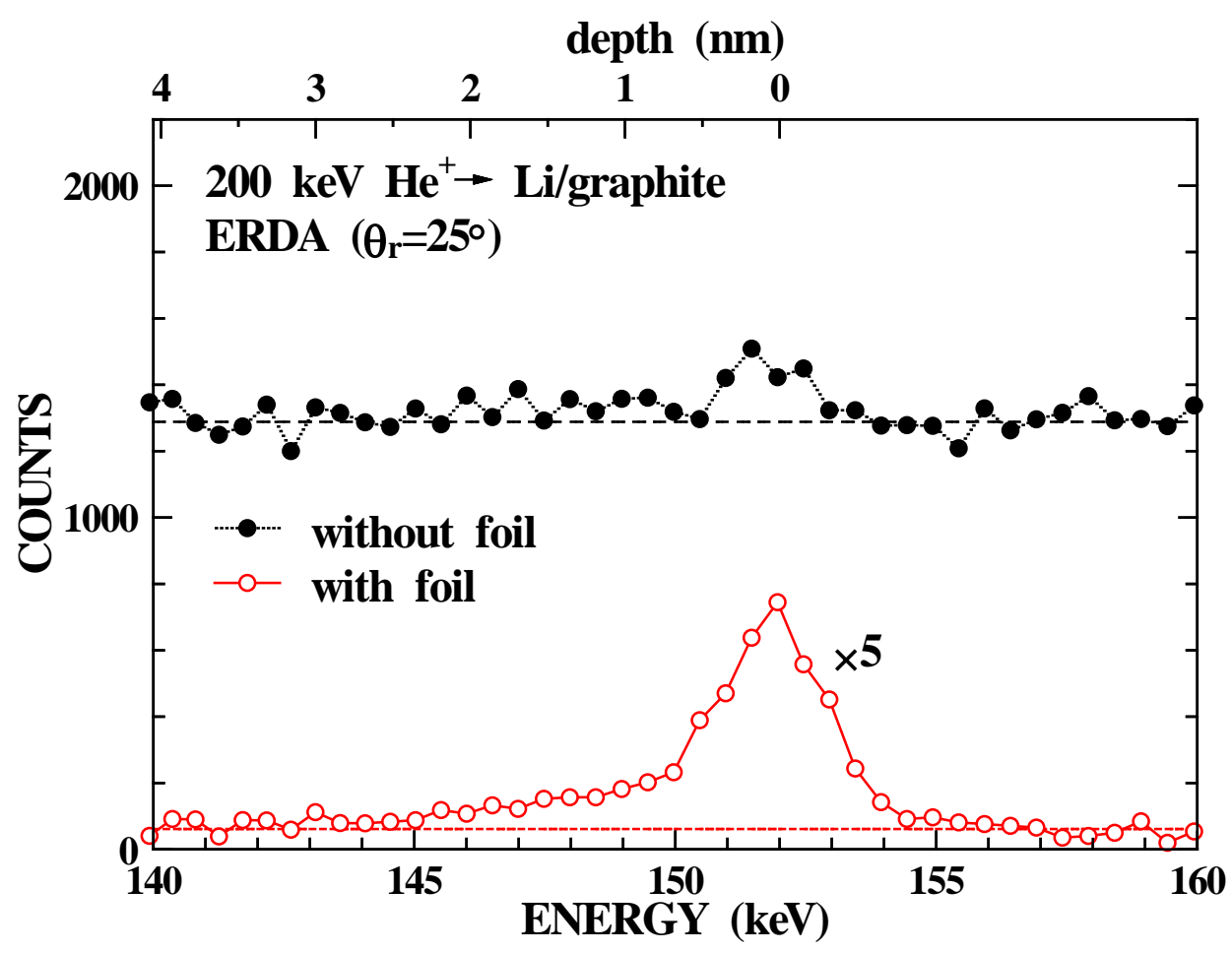

Fig. 2 


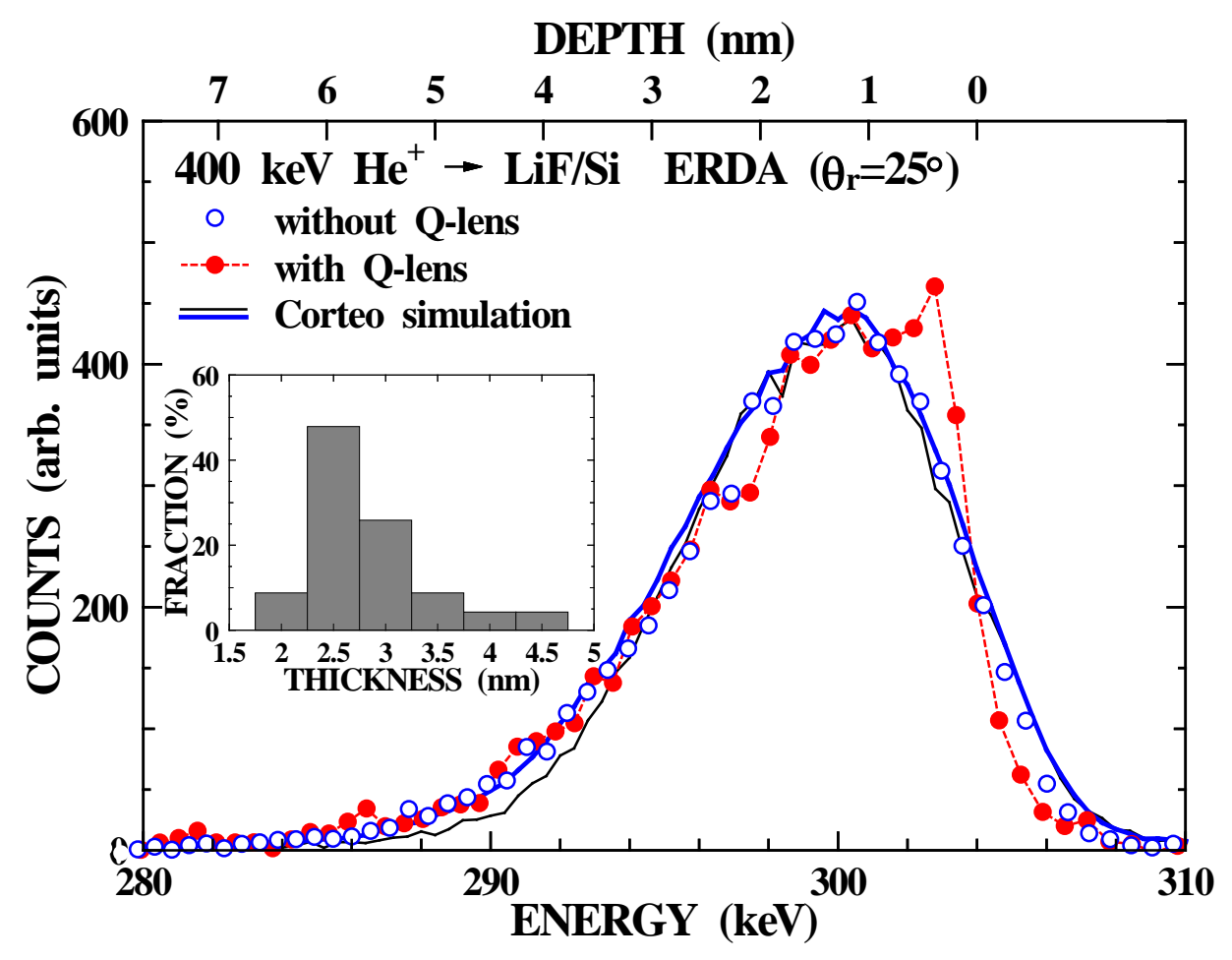

Fig. 3

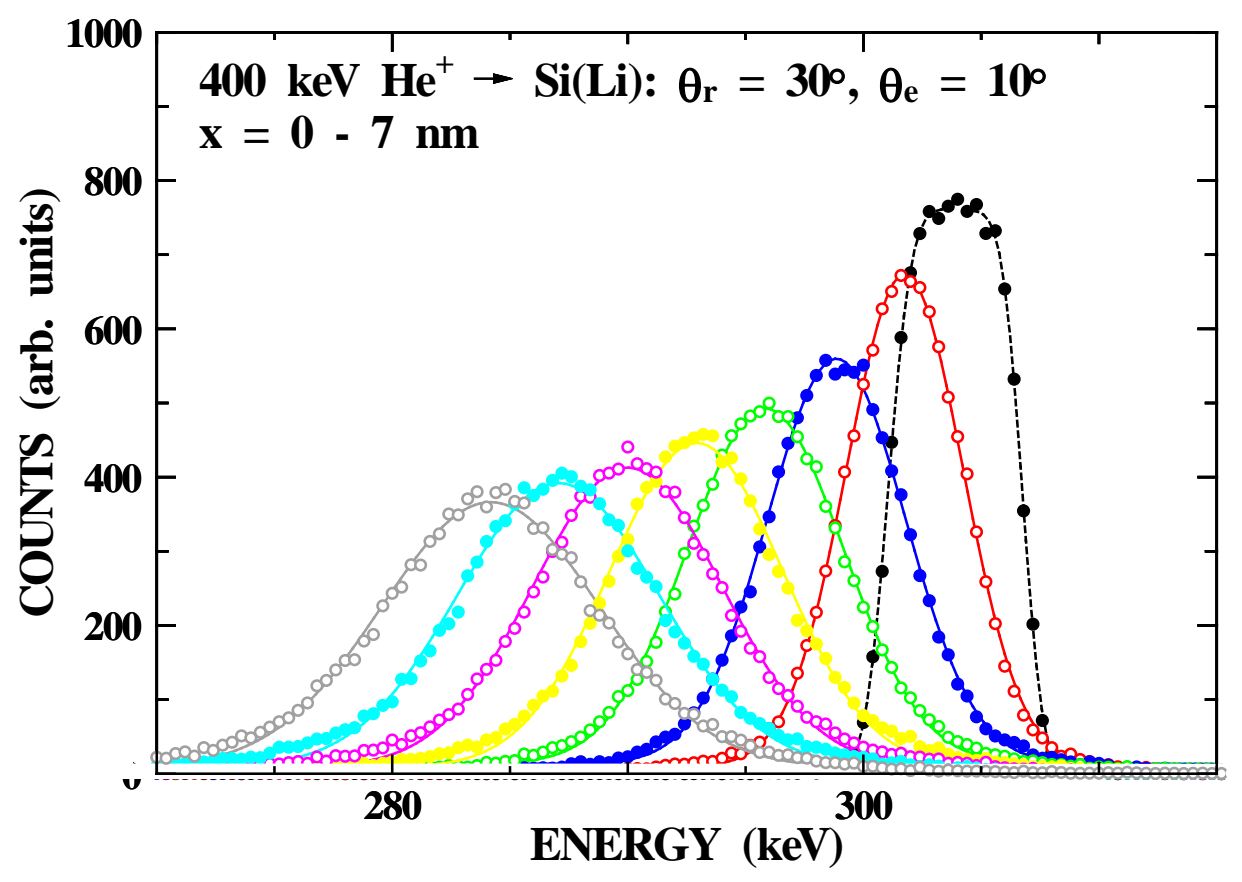

Fig. 4 


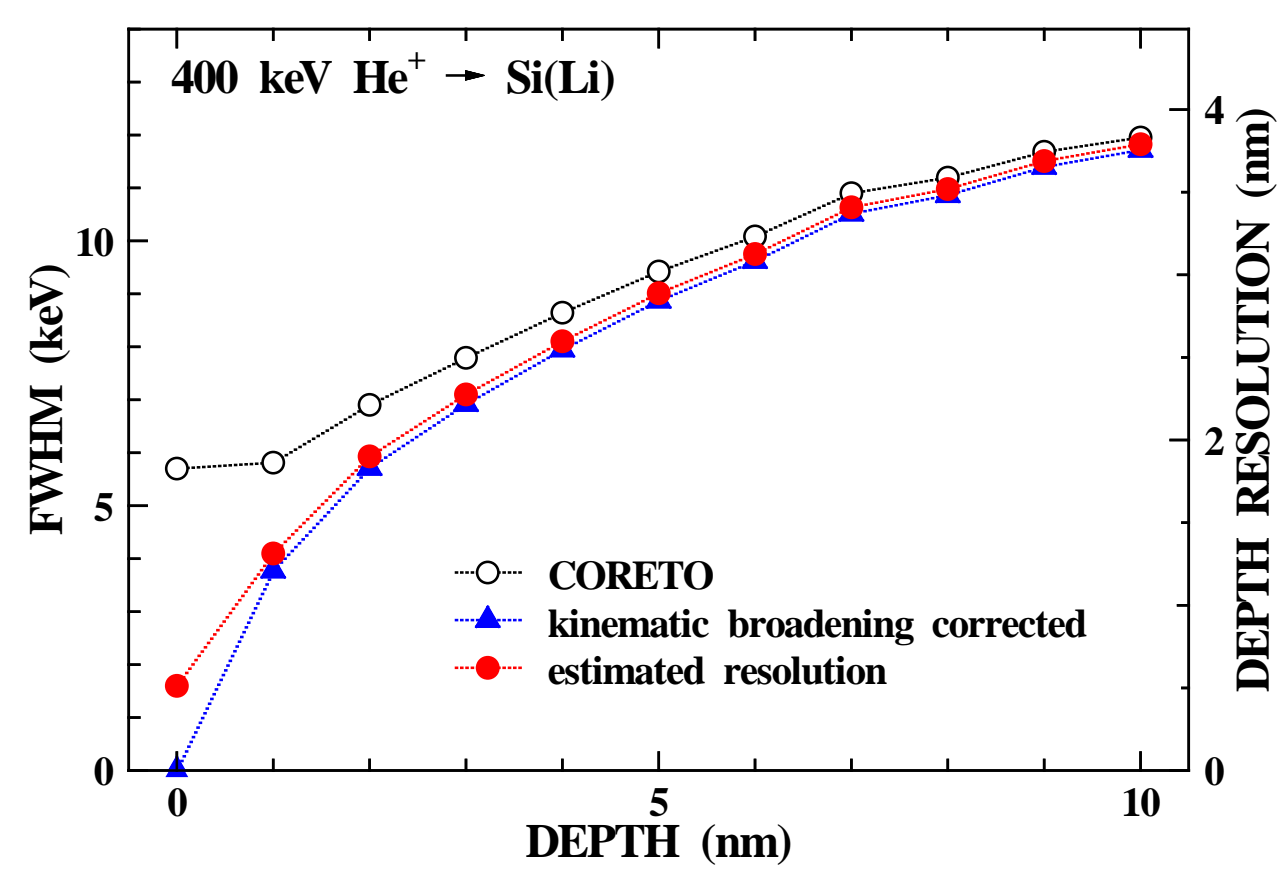

Fig. 5 